\title{
La controverse du « gaz de charbon » en France (2006-2018) : conflits de nomination et mise en question de la neutralité de l'expertise
}

The controversy over "coal gas" in France (2006-2018): naming conflicts and the questioning of expertise neutrality

La controversia del "gas de carbón» en Francia (2006-2018): conflictos de designación y el cuestionamiento de la neutralidad de los conocimientos especializados

\section{Marieke Stein}

\section{(2) OpenEdition}

\section{Journals}

Édition électronique

URL : https://journals.openedition.org/mots/24390

DOI : $10.4000 /$ mots. 24390

ISSN : 1960-6001

Éditeur

ENS Éditions

\section{Édition imprimée}

Date de publication : 5 mars 2019

Pagination : 69-85

ISBN : 979-10-362-0130-1

ISSN : 0243-6450

Référence électronique

Marieke Stein, «La controverse du « gaz de charbon » en France (2006-2018) : conflits de nomination et mise en question de la neutralité de l'expertise ", Mots. Les langages du politique [En ligne], 119| 2019, mis en ligne le 01 janvier 2022, consulté le 23 avril 2022. URL : http://journals.openedition.org/ mots/24390 ; DOl : https://doi.org/10.4000/mots.24390

\section{(C) ENS Éditions}




\section{La controverse du " gaz de charbon " en France (2006-2018) : conflits de nomination et mise en question de la neutralité de l'expertise}

L'analyse des désignations et de la valeur argumentative du lexique a déjà donné lieu à maints fructueux articles dédiés à l'étude des fonctionnements discursifs et des «enjeux argumentatifs de la nomination " (Amossy, Koren, 2010, p.16) dans les champs politiques ou sociétaux. Ont été étudiés, par exemple, les lexèmes «communauté » (Lecolle, 2007), «camp de concentration» (Krieg-Planque, 2000), « développement durable» (Krieg-Planque, 2010), "génocide» (Angenot, 2014) et, plus largement, le lexique des discours xénophobes contemporains (Breton, 1997). S'inscrivant dans les mêmes perspectives de recherche, la présente contribution s'intéresse toutefois à un autre champ, le champ techno-scientifique. Dans les domaines scientifique, technique et industriel, le lexique, qui emprunte souvent à des «langues de spécialité», pourrait sembler régulé par la science, déterminé par une rationalité plus grande, un rapport au réel plus immédiat, et donc ne pas présenter la même «épaisseur» ni la même polysémie que le vocabulaire courant qui parcourt et structure l'espace public. Pourtant, comme l'a montré Sezin Topçu, l'énergie n'échappe pas à la politisation du lexique, et dans ce domaine aussi les mots se révèlent être «des instruments majeurs de gouvernementalité» (Topçu, 2013, p. 196).

La présente étude s'appuie sur l'analyse au long cours d'une controverse environnementale active depuis quelques années dans le nord et l'est de la France, et qui concerne des forages exploratoires de «gaz de couche de charbon ». Cet hydrocarbure non conventionnel, voisin du gaz de schiste, n'a encore jamais été exploité sur un territoire francophone, et a donc fait son entrée dans la langue française en 2004 (année des premières demandes de permis d'exploration) par la traduction littérale de sa dénomination anglophone, «Coal Bed Methane» (CBM). Pour reprendre la distinction rappelée par Roselyne Koren entre dénomination (association référentielle durable, constante et conventionnelle, entre un objet et un signe) et désignation (qualification employée 
en discours, non stable et non consensuelle, voir Koren, 2016, p. 3-4), quatorze ans après son arrivée en France, ce gaz n'a toujours pas de dénomination stabilisée. Seules des désignations éphémères émergent au fil des discours produits par les acteurs industriels, institutionnels, médiatiques ou associatifs : "gaz de couche », "gaz de houille », "gaz de charbon » et même "grisou ». Ces désignations sont au cœur d'une vive «controverse d'étiquetage» (Angenot, 2014) entre «pros» et «antis».

Quelle peut être la charge émotionnelle ou affective, la valeur argumentative d'un mot désignant un hydrocarbure? Quelle différence entre «méthane », « gaz naturel» et «grisou » ? Sous une apparence de scientificité, un conflit de nomination se joue ici, qui met en lumière l'antagonisme entre des représentations concurrentes de l'environnement et qui comporte une forte dimension politique, qu'il s'agisse pour les industriels d'influer sur des décisions politiques (pour l'obtention de subventions ou l'assouplissement du cadre réglementaire) ou d'obtenir l'acceptabilité sociale de cette énergie. Cette notion d'acceptabilité sociale, venue du contexte anglo-saxon, est complexe et souvent mal définie (Fortin, Fournis, 2014). La recherche en sciences humaines et sociales tend à y voir un processus de négociation sociale destinée à aboutir à un consensus autour de projets contestés, plutôt que l'obtention d'un consentement des populations par les stratégies de communication et de relations publiques des promoteurs; il n'en reste pas moins que la notion, comme l'écrit Corinne Gendron, reste «teintée par la volonté des décideurs de mieux maîtriser les contestations dont leurs décisions peuvent faire l'objet» (Gendron, 2014, p. 122). Le lien entre les notions d'acceptabilité et de gouvernementalité est essentiel dans la présente étude.

L'analyse proposée dans ces pages vise à montrer que, loin d’être régis par une rationalité supérieure, les choix lexicaux dans le domaine des hydrocarbures ont une forte dimension axiologique et sont au cœur de stratégies discursives visant l'acceptabilité sociale de cette nouvelle énergie, dans le cadre d'une communication dite "sensible» (Libaert, 2009). Elle éclaire les enjeux stratégiques et politiques de ce conflit de nomination, dont l'un des effets est la neutralisation des oppositions. En effet, alors que le gaz de schiste, hydrocarbure de la même famille géologique, très proche par ses techniques d'extraction et ses impacts environnementaux, a occasionné et occasionne encore, en France et dans le monde, des controverses médiatisées et des mobilisations massives, le CBM, lui, suscite une moindre opposition, malgré des impacts environnementaux bien documentés (Bonijoly et al., 2013). Suivre l'apparition, les usages, la fortune de ces différentes désignations apporte un éclairage sur les étapes de l'appropriation étatique et sociétale d'une énergie nouvelle, et un outillage méthodologique intéressant pour l'analyse des controverses.

Ce travail s'appuie sur des analyses quantitatives et qualitatives réalisées à partir de textes qui couvrent l'ensemble de la période concernée par les projets de forages de CBM en Lorraine, soit 2006-2018. Ces textes ont été regroupés en 
trois catégories. D’abord, les productions émanant du promoteur du projet d'extraction, et qui comprennent aussi bien ses supports de communication externe (une douzaine de communiqués de presse et autant de supports de communication diffusés dans la zone géographique concernée par les forages) que les documents administratifs déposés aux différentes étapes de son projet (douze dossiers de demande d'autorisation d'ouverture de travaux miniers et notices d'impact). Ensuite, onze rapports techniques et scientifiques (comme la Synthèse sur les gaz de houille de l'INERIS'1 et du BRGM²), gouvernementaux (les rapports commandés par le CGIET3 puis l'OPECST4 en 2012 et 2013), ou réalisés par des organismes interprofessionnels comme l'Institut français du pétrole Énergies nouvelles (IFPEN) et l'Agence internationale de l'énergie (IEA). Ces rapports, les seuls disponibles en langue française sur le CBM, constituent des «sommes» d'information sur cet hydrocarbure et jalonnent le développement de la controverse. Enfin, un corpus de presse de 450 articles (majoritairement issus de la presse quotidienne régionale $[\mathrm{PQR}]$ lorraine) publiés entre 2006 (première mention du projet d'extraction dans la presse) et août 2018 (clôture de notre corpus), et couvrant donc une période de douze ans propice à l'étude de l'évolution du traitement médiatique du projet lorrain d'extraction de CBM. Tous ces documents ont d'abord été l'objet d'analyses quantitatives (grâce au logiciel Atlas.ti) afin de mettre en évidence les désignations privilégiées. Ces relevés ont ensuite été affinés par des analyses qualitatives empruntant majoritairement à la rhétorique et à l'argumentation. Cette étude ne néglige pas les éléments du contexte (territorial, social, politique, institutionnel, etc.), nécessaires à la compréhension des effets persuasifs des discours étudiés et à la mise en évidence des enjeux de ces désignations dans une stratégie d'acceptabilité. Elle aboutira à préciser les axiologies à l'œuvre derrière les choix de dénominations, pour finalement interroger la réalité de la neutralité affirmée par certains des experts au regard des désignations qu’ils mobilisent.

\section{Le Coal Bed Methane : une énergie nouvelle aux désignations changeantes}

Lorsqu'en 2004 une société australienne, Kimberly Oil (qui deviendra en 2006 European Gas Limited [EGL]), dépose ses premières demandes de permis exclusifs de recherches (PER) de CBM en France, cet hydrocarbure dit «non conventionnel» (car captif de couches géologiques trop difficiles d'accès pour permettre son extraction par des moyens « conventionnels») est encore inconnu en France.

1. Institut national d'évaluation des risques scientifiques et technologiques.

2. Bureau de recherches géologiques et minières.

3. Conseil général de l'industrie, de l'énergie et des technologies.

4. Office parlementaire d'évaluation des choix scientifiques et technologiques. 
Exploité depuis peu aux États-Unis et en Australie, il n'est d'abord désigné en France que par sa dénomination anglophone, Coal Bed Methane, accompagnée de son acronyme (CBM) et de sa traduction littérale, "gaz de couche de charbon ». Lorsque les premiers puits d'exploration sont forés en 2006 et 2008, la presse régionale leur consacre quelques articles, en désignant généralement ce gaz par le terme générique "gaz» sans en spécifier la nature. Quant aux professionnels de la filière, ils utilisent à cette époque l'acronyme anglais CBM, parfois traduit par « gaz de couche» ou " gaz de couche de charbon », à l'instar du rapport 2008 de l'IFP Énergies nouvelles, où CBM et Coal Bed Méthane représentent $88 \%$ des désignations utilisées (Maisonnier, 2008, p.2).

C'est en 2012 que ces dénominations techniques vont céder la place à d'autres : «gaz de houille », d'abord, qui va largement s'imposer dans la presse et dans les rapports professionnels et gouvernementaux; "grisou », ensuite, souvent accompagné du même développement sur sa dangerosité, repris littéralement ou presque, dans la presse : "Jadis hantise des mineurs à l'origine des meurtriers coups de grisou, le gaz de houille [...] pourrait être une solution pour la reconversion économique de cette région » (AFP, 28 janvier 2013); «Longtemps cauchemar des mineurs, le grisou est en passe d’être réhabilité » (Sciences et Avenir, novembre 2014, n813)...

Que s'est-il passé entre ces deux périodes aux désignations si différentes? Quel est l'événement qui détermine ce changement entre une phase «scientifique » de la dénomination, et les désignations fautives de l'après-2011? Car les désignations qui dominent ensuite ne sont pas exactes scientifiquement. En effet, le gaz de houille désigne depuis lexıxe siècle un gaz manufacturé, issu de la combustion du charbon (Maisonnier, 2008). Quant au grisou, s’il partage avec le CBM sa nature chimique (du méthane) et son origine géologique (le charbon), il désigne un « gaz combustible qui se dégage spontanément dans les mines de houille» (Le Robert). Grisou est donc le synonyme, dans la langue vernaculaire, de gaz de mine, ce gaz accumulé dans les galeries de mines au cours du processus d'extraction du charbon, ou après l'arrêt de son exploitation. Le CBM, lui, reste captif de couches charbonneuses profondes et non exploitées. Ne rencontrant pas d'oxygène, il n'est pas dangereux, à la différence du grisou. Avec des méthodes d'extraction et des impacts environnementaux très différents, les deux gaz relèvent d'ailleurs d'une classification différente. Pour les professionnels des hydrocarbures, le grisou (ou gaz de mine) est dit « conventionnel », tandis que le CBM est «non conventionnel », car récupérable par des techniques «non conventionnelles» (forages horizontaux généralement associés à la fracturation hydraulique).

Nous sommes donc passés en 2010-2011 de désignations à vocation scientifique à des désignations que l'on pourrait qualifier de «pseudo-scientifiques». Ou, pour se situer sur le terrain de l'analyse discursive, d'un régime de désignation à vocation descriptive à un régime de désignation à vocation persuasive. 
L'événement qui opère cette bascule est la très médiatisée controverse du gaz de schiste. En 2010 et 2011, une mobilisation éclair de citoyens et d'élus concernés par des permis de recherche de gaz de schiste dans le Sud, renforcée par le soutien de personnalités médiatiques (dont José Bové), aboutit en juillet 2011 à l'adoption rapide de la loi Jacob interdisant la fracturation hydraulique, loi qui met (provisoirement) fin aux projets d'exploration de gaz de schiste en France (voir Chateauraynaud, Debaz, 2011; Chailleux, 2015). La forte médiatisation de cette controverse, qui fait du gaz de schiste un problème public, devait nécessairement compromettre l'acceptabilité des projets de l'entreprise EGL qui développait en toute quiétude des projets de forages de CBM en Lorraine, dans le Nord-Pas-de-Calais, à Gardanne, Saint-Étienne et Lons-le-Saunier. Si, jusqu'à cette controverse, la société n’avait pas vraiment caché le possible recours à la fracturation, - une géologue travaillant sur l'un des forages confiait même en 2008 à un journaliste : "nous utilisons des techniques de forage horizontal et de fracturation hydraulique» (La Semaine numérique $5,1^{\mathrm{er}}$ août 2008) -, il devenait urgent, après la loi Jacob, de récuser l'emploi de cette technique. De fait, contrairement aux exploitations de gaz de schiste qui nécessitent systématiquement la fracturation de la roche-mère, certains gisements de CBM peuvent être exploités par simple pompage, du moins en début d'exploitation (la plupart nécessitant la fracturation hydraulique à un stade plus avancé de l'exploitation, dans un souci de rentabilité). La nature même du gaz recherché autorisait donc une communication axée sur le non-recours à cette technologie controversée. Après une période de quasi-absence médiatique de cette société au moment de la crise du gaz de schiste, de mi-2010 à fin 2011, un changement de stratégie communicationnelle s'amorce à l'été 2012. Cette nouvelle stratégie consiste en grande partie, pour l'entreprise et pour les grands acteurs français de l'énergie auxquels elle est liée (au premier rang desquels l'Union française des industries pétrolières [UFIP]), à imposer de nouvelles dénominations pour l'énergie recherchée, et à créer ainsi une confusion propre à favoriser, sinon l'adhésion des populations concernées à son projet, du moins la suspension de leur jugement.

\section{Stratégie du flou et communication d'acceptabilité : les effets argumentatifs et les connotations mémorielles des désignations choisies}

À partir de l'été 2012, EGL déploie une stratégie discursive en deux volets. Le premier consiste simplement à dissocier le CBM du gaz de schiste en multipliant dans la presse les déclarations de non-utilisation de la fracturation

5. La Semaine numérique est le site internet de l'hebdomadaire lorrain La Semaine. 
hydraulique. Le second consiste à imposer dans l'espace public de nouvelles désignations du gaz recherché, grâce à des amalgames relevant d'une manipulation des faits et des affects (voir notamment Breton, 1997) et au choix d'une référenciation subjective à forts enjeux argumentatifs.

D’abord, il s'agit de faire de cette énergie étrangère, inconnue en France - donc potentiellement inquiétante -, une énergie familière et rassurante. Pour cela, le terme "gaz de houille» est idéal : dans un bassin houiller lorrain douloureusement marqué par la fermeture de son dernier puits charbonnier en 2004, les connotations de ce terme sont la familiarité, la continuité historique, le rappel d'une prospérité passée... Ensuite, « gaz de houille » désignant en réalité un gaz qui résulte de la transformation du charbon en coke, l'expression dénote un gaz «qui ne coûte rien», qui est lui-même le produit dérivé d'une autre industrie, et qui n'a aucun impact écologique : un gaz entièrement positif, une énergie quotidienne, propre et à bas coût. Ce travail sur la familiarité et la proximité prend davantage d'ampleur encore avec l'assimilation du CBM avec le « grisou », par un amalgame permettant d'activer à la fois la valeur argumentative et les connotations affectives et mémorielles du grisou dans le bassin houiller. Dans la langue vernaculaire, le « grisou » est un gaz dangereux pour les mineurs. À force de voir répétée, notamment dans la PQR, l'identité entre «gaz de houille » (carCBM n'apparaît jamais) et " grisou » (terme employé dans 62 \% des articles de la PQR sur le sujet à partir de 2012), un glissement interprétatif s'opère chez les lecteurs, qui peut être résumé par un paralogisme, dont la première prémisse est fausse et entraîne une conclusion fausse : "Le gaz de houille c'est du grisou. Le grisou doit être capté pour ne pas exploser. Donc il faut capter le gaz de houille.»

En réalité, le terme "grisou» est impropre, car le CBM ne s'accumule pas dans des «vides » et ne fait courir de risques à personne. Cet amalgame constitue pourtant un axe majeur de la communication du groupe EGL, comme en témoigne le chapeau du publi-communiqué paru le 4 mars 2016 dans Le Républicain lorrain :

C'est dans une démarche résolument prospective que la Française de l’Énergie6 a pour objectif d'exploiter le gaz de houille contenu en grande quantité dans le sous-sol lorrain. Vous avez dit gaz de houille? Un sujet qui fascine autant qu'il inquiète. L'histoire de la Lorraine s'est construite en partie sur le grisou : si le charbon a fait la richesse de la région dans le passé, le gaz de charbon, lui, peut devenir sa richesse de demain.

Ce court extrait suffit à illustrer cette rhétorique fondée en grande partie sur l'allusion : le texte ne dit pas que le gaz recherché est le grisou, mais pousse le lecteur à cette interprétation par l'emploi du verbe «inquiéter». Il faut noter d'ailleurs qu'en quelques lignes, trois désignations sont utilisées : gaz de

6. Nouveau nom de la société EGL depuis juillet 2015. 
houille, grisou, gaz de charbon... Induire la confusion est une stratégie permettant d'amener le lectorat à renoncer à comprendre précisément de quelle énergie il s'agit et quelle sera la technique employée, pour le ramener à ses affects (par le lexique de la fascination, des émotions, de l'intérêt matériel) et le rassurer avec un lexique familier. "Grisou» fonctionne alors comme un de ces «mots piégés» dont Andreas Freund écrivait qu’ils

ne sont rien d'autre que de la mésinformation véhiculée par des vocables au contenu tendancieux. Une fois passés dans le langage courant avec leur charge de sens démagogique, ils serviront de munitions de petit calibre dans la bataille permanente qui se livre pour la conquête des esprits. (Freund, 1991, p.151)

\section{" Gaz de charbon ", une tentative de clarification institutionnelle?}

La désignation "gaz de charbon » apparaît à la fin des années 2000 dans les textes des industriels des énergies fossiles, mais elle reste très marginale. Pourtant, en 2015, le Journal officiel de la République française publie une mise à jour de son «vocabulaire du pétrole et du gaz» qui fixe les traductions officielles de termes étrangers. Dans ce document, Coal Bed Methane, entré dans la terminologie officielle en 2009 sous la traduction "méthane houiller», est désormais traduit « gaz de charbon ». Aussitôt, les promoteurs gaziers et pétroliers généralisent et diffusent cette dénomination nouvelle, qui fait son entrée dans la presse régionale au cours de l'année 2016.

L'analyse des usages de ce syntagme en contexte discursif révèle qu'il bénéficie des mêmes connotations mémorielles et affectives que "gaz de houille» ou « grisou » (proximité, utilité, prospérité passée). Scientifiquement correcte (puisque le Coal Bed Methane est captif du charbon), l'expression est néanmoins suffisamment englobante pour permettre des glissements de sens : si, pour les spécialistes, elle équivaut à «Coal Bed Methane», en revanche pour les journalistes et le grand public elle désigne aussi bien le gaz de mine que le CBM. Cette ambiguïté était déjà présente dans les textes des acteurs des énergies fossiles, à l'instar de l'IFPEN qui, dans Hydrocarbures de roche-mère : état des lieux, définissait ainsi le "gaz de charbon»:

Le gaz de charbon est également une importante ressource non conventionnelle exploitée dans de nombreux pays. [...] En France, European Gas dispose de plusieurs permis dans le Nord-Pas-de-Calais (Gazonor), en Lorraine, autour de LonsLe-Saunier et au nord de Marseille. Avec $136 \mathrm{Gm}^{3}$ de ressources estimées, seul Gazonor est en phase de production. (Vially et al., 2013, p. 57)

L'IFPEN assimile sous un même vocable le gaz de mine (seul gaz produit par Gazonor) et le Coal Bed Methane, objet des permis de recherche des trois autres secteurs cités... 
Il faut signaler que, dans le collège «énergies fossiles » de la Commission d'enrichissement de la langue française, sur 17 " experts » chargés de proposer des traductions au vocabulaire anglo-saxon des hydrocarbures, dix sont directement issus de l'industrie pétrolière, dont six sont des salariés, consultants ou anciens responsables de Total (les sept autres membres de la commission étant des linguistes). On peut donc supposer que la terminologie officielle a été proposée par l'industrie pour favoriser la neutralisation des controverses futures en choisissant un terme de désignation suffisamment imprécis et ouvert pour éviter l'exposé clair des conditions d'exploitation d'un hydrocarbure largement contesté dans d'autres pays. D'un point de vue linguistique, le syntagme «gaz de charbon » est suffisamment plastique pour pouvoir être convoqué dans les textes de communication organisationnelle, voire institutionnelle, comme hyperonyme de deux gaz dont le seul dénominateur commun est la nature de leur matrice géologique, mais que toutes leurs autres caractéristiques opposent (mode de présence dans la roche et surtout techniques d'extraction : caractère conventionnel ou pas, difficultés opérationnelles, impacts environnementaux, etc.).

Le «contrôle des mots» (Topçu, 2013) est ici imposé non par le pouvoir politique, mais par les industriels soucieux de pouvoir développer une filière contestée, avec l'acceptation plus ou moins passive, plus ou moins consciente, d'acteurs politiques et institutionnels. Notre hypothèse est que, lors des discussions du comité «énergie » de la Commission d'enrichissement de la langue française, les représentants du ministère et les linguistes ont cherché, conformément à leur mission, une clarification de la langue, alors que les « experts» en géosciences, tous plus ou moins affiliés au grand groupe pétrolier qui a d'ailleurs financé le Lexique distribué à l'issue des travaux de la commission, ont cherché l'obscurcissement. «Gaz de charbon » réunissait parfaitement les deux intentions : un mot simple; une réalité englobante, favorisant les amalgames stratégiques.

\section{Du côté des experts, quelle axiologie pour le lexique?}

L'étude des désignations utilisées dans la controverse autour du CBM peut contribuer à une catégorisation des différents acteurs impliqués. «Gaz de couche » signale clairement les opposants, qui privilégient nettement cette désignation et relient ainsi le CBM à l'autre «gaz de couche » qu'est le gaz de schiste, rappelant ainsi les forts enjeux environnementaux du CBM. «Gaz de houille», popularisé par les promoteurs, est finalement devenu le terme courant, peu marqué axiologiquement. "Grisou » et «gaz de charbon » signalent les promoteurs du CBM, qui font généralement de ces termes un usage abusif, en exploitant leur caractère vernaculaire (pour «grisou») et englobant (pour 
"gaz de charbon ») afin d'entretenir une confusion entre le gaz réellement objet des explorations et le "gaz de mine» (un gaz facile d'accès dont l'exploitation est utile pour sécuriser les anciennes mines et réduire l'échappée du grisou dans l'atmosphère).

À partir de ce constat, le relevé des désignations utilisées dans les rapports d'experts permet-il de déterminer leur positionnement axiologique vis-à-vis du problème sociotechnique qu'ils sont supposés éclairer? Dans une controverse, des «experts» sont régulièrement convoqués pour éclairer la décision politique. Si, dans les débats sur l'énergie, ceux-ci sont souvent en lien avec les opérateurs, ce sont aussi parfois des géologues ou des chercheurs présentés comme «indépendants». Dotés de connaissances reconnues, ils interviennent sous couvert de neutralité dans le but d'éclairer l'opinion des décideurs.

Dans le cadre de la présente étude sur les désignations françaises du Coal Bed Methane, la scission des termes entre deux pôles sémantiques, qui sont aussi deux pôles axiologiques, est si nette qu'il est tentant d'utiliser cette approche pour tenter de déterminer où se situent les experts, en fonction des désignations du CBM qu'ils privilégient. Leur neutralité et la rationalité affirmée de leur approche devraient impliquer, au minimum : 1) un accord sur une terminologie stable correspondant à des référents précis et identifiés; 2 ) le recours aux termes les plus précis (du type: CBM, gaz de couche de charbon); 3) le non-recours aux termes impropres (grisou) ou englobants (gaz de charbon), sauf à clarifier le référent par une explicitation métalinguistique. Nous avons donc recensé les termes utilisés par les experts, et analysé leur fonctionnement en discours (tableau 1).

\begin{tabular}{|c|c|c|c|c|c|c|c|c|}
\hline & & & \begin{tabular}{|c|} 
CBM / Gaz \\
de couche de \\
charbon
\end{tabular} & $\begin{array}{l}\text { gaz de } \\
\text { houille }\end{array}$ & $\begin{array}{l}\text { gaz de } \\
\text { charbon }\end{array}$ & grisou & autres & total \\
\hline \multicolumn{9}{|c|}{ Pour comparaison : désignations utilisées par l'opérateur } \\
\hline \multirow{3}{*}{ EGL } & 2006-2017 & $\begin{array}{c}\text { Documents techniques et réglementaires (demandes de } \\
\text { permis, programmes de travaux...) (12) }\end{array}$ & 38,5 & 40,6 & 10,1 & 2 & 8,8 & 100 \\
\hline & $2016-2018$ & Communiqués de presse (12) & 0 & 11,3 & 29 & 0 & 59,7 & 100 \\
\hline & 2004-2017 & $\begin{array}{l}\text { Présentations, plaquettes, lettres d'information (7) } \\
\text { diffusées dans le bassin houiller lorrain }\end{array}$ & 0 & 3,7 & 7,4 & 40,8 & 48,1 & 100 \\
\hline \multicolumn{9}{|c|}{ Rapports d'acteurs de l'énergie } \\
\hline IFPEN & 2008 & $\mathrm{CBM}$, bilan et perspectives & 88 & 0 & 12 & 0 & 0 & 100 \\
\hline IFPEN & 2012 & $\begin{array}{l}\text { Les hydrocarbures non conventionnels : évolution ou } \\
\text { révolution? }\end{array}$ & 41,7 & 50 & 0 & 8,3 & 0 & 100 \\
\hline IAE & 2012 & Des règles d'or pour un åge d'or du gaz & 3,2 & 96,8 & 0 & 0 & & 100 \\
\hline Mirova/natixis & 2012 & $\begin{array}{l}\text { Gaz de schiste ct autres gaz non-conventionnels: } \\
\text { nouvelles ressources, nouveaux enjeux }\end{array}$ & 21,4 & 2,4 & 76,2 & 0 & 0 & 100 \\
\hline IFPEN & 2013 & Hydrocarbures de roche-mère : état des lieux & 25 & 23,7 & 51,3 & 0 & 0 & 100 \\
\hline \multicolumn{9}{|c|}{ Rapports gouvernementaux (commandes) } \\
\hline CGIET-CGEDD & 2012 & Les hydrocarbures de roche-mère en France & 57,2 & 28,5 & 0 & 14,3 & 0 & 100 \\
\hline OPECST & 2013 & $\begin{array}{c}\text { Rapport sur les techniques alternatives à la fracturation } \\
\text { hydraulique pour l'exploration et l'exploitation des } \\
\text { hydrocarbures non conventionnels }\end{array}$ & 33,4 & 65 & 0 & 1,6 & 0 & 100 \\
\hline INERIS/CORE & 2013 & $\begin{array}{l}\text { Maîtrise des risques environnementaux liés à la filière } \\
\text { « gaz de houille } ~\end{array}$ & 0 & 96,7 & 3,3 & 0 & 0 & 100 \\
\hline INERIS/BRGM & 2013 & Synthèse sur les gaz de houille & 56 & 35,3 & 8,2 & 0,5 & 0 & 100 \\
\hline \multicolumn{9}{|c|}{ Rapports de recherche universitaire } \\
\hline \multirow{2}{*}{$\begin{array}{l}\text { CNRS / Université } \\
\text { de Lorraine }\end{array}$} & 2014 & Présentation du projet $\alpha$ Gazhouille » & 28 & 24 & 48 & 0 & 0 & 100 \\
\hline & 2016 & $\begin{array}{l}\text { Le projet d'exploitation du gaz de charbon en Moselle et } \\
\text { son intégration dans le territoire }\end{array}$ & 17,9 & 18,4 & 51,9 & 5,7 & 6,1 & 100 \\
\hline & & Total & 27,4 & 39,8 & 19,8 & 4,9 & 8,8 & 100 \\
\hline
\end{tabular}

Tableau 1. Désignations utilisées pour le CBM dans le corpus « rapports» (en \%) 
Parmi les onze rapports examinés, plusieurs émanent d'organismes professionnels favorables au développement des hydrocarbures non conventionnels (IFPEN, IEA, Mirova). Dans ce type de rapports, les scientifiques sollicités sont généralement des géologues ou des ingénieurs des mines liés aux entreprises pétrolières, dont le positionnement est explicite ; les termes les plus usités (après 2012) sont donc, sans surprise, "gaz de houille» et "gaz de charbon », avec les ambiguïtés et glissements qu'ils favorisent. II ne faudrait pas négliger toutefois le facteur temporel : le document de l'IFPEN daté de 2008 (donc antérieur à la controverse du gaz de schiste et à la loi Jacob) utilise en grande majorité «CBM» (88\% des occurrences), alors que «gaz de houille» et «gaz de charbon » s'imposent après 2011. Au sein d'une même institution (l'IFPEN), les désignations s'ajustent en permanence en fonction des impératifs communicationnels des acteurs de la profession.

D’autres rapports se présentent comme issus d'une expertise indépendante, dédiée à éclairer le débat public par l'apport d'une information aussi complète que possible sur les différents aspects techniques, réglementaires, économiques et sociétaux liés à cette énergie nouvelle. Trois rapports correspondent à cette visée : celui du CGIET et du CGEDD (CGIET, CGEDD, 2012), celui de l'OPECST (Lenoir, Bataille, 2013) et la Synthèse sur les gaz de houille de l'INERIS et du BRGM (Bonijoly et al., 2013). Le premier privilégie «Coal Bed Methane» ou «CBM» $(57,2 \%$ des occurrences) puis «gaz de houille» $(28,5 \%)$, et enfin " grisou » (14,3\%). Outre l'emploi majoritaire de la désignation la plus précise et la plus exacte, le rapport affiche le souci de clarifier les termes employés, comme «gaz de houille», dont une note de bas de page précise :

À ne pas confondre avec le "gaz de mines», également du grisou, que l’on récupère par simple pompage dans les anciens travaux miniers non encore ennoyés, par exemple en France dans l'ancien bassin houiller du Nord-Pas-de-Calais. (CGEIT, CGEDD, 2012, p. 14)

De même, le rapport de l'OPECST clarifie les désignations employées, et privilégie la plus exacte (CBM) et la plus courante (gaz de houille). Quant à l'étude française de référence sur le sujet, la Synthèse sur les gaz de houille, elle témoigne d'un souci constant de bien distinguer entre des énergies apparemment proches, et de préciser au maximum leurs caractéristiques chimiques, techniques et géologiques. Là encore, la dénomination «CBM/Coal Bed Methane» est majoritaire (56\% des occurrences), suivie par le terme usuel "gaz de houille» $(35,3 \%)$ puis "gaz de charbon» $(8,2 \%)$. Le terme "grisou» apparaît, mais entre guillemets et assorti d'un commentaire métalinguistique présentant ce terme comme relevant de l'usage courant, et non scientifique. Dès le début, le CBM est distingué du gaz de mine par un métadiscours qui montre la rigueur de ce rapport en matière de terminologie : «La présente note est centrée sur le gaz de houille et dans ce qui suit nous utiliserons indifférem- 
ment les termes gaz de houille ou CBM.» (Bonijoly et al., 2013, p.15).

Une première constatation se dessine à ce stade : les désignations, au cœur même de la sphère experte, ne concordent pas et ne sont pas stabilisées. Les rapports hésitent tous entre différentes désignations. Le point commun à ces premiers rapports est toutefois l'explicitation des référents : les auteurs commentent et justifient leurs choix terminologiques, et présentent bien de ce fait les termes proposés comme des désignations non fixées, reconnaissant ainsi la nouveauté, voire l'étrangeté, d'un gaz qui n'a pas de traduction française définitive. A contrario, le discours des promoteurs impose des dénominations sous forme assertive ("Ce gaz non conventionnel n'est autre que le fameux "grisou" qui effrayait tant les mineurs", Challenges, 24 mai 2016), comme si la désignation imposée était objet d'un consensus - manière usuelle en rhétorique de nier toute ambiguïté et, partant, de désamorcer toute controverse.

Seconde constatation : les documents techniques et les rapports gouvernementaux, dans leur majeure partie, n’emploient pas «grisou » pour désigner le CBM, le terme étant non technique et impropre. EGL même, dans ses demandes de permis et autres dossiers techniques destinés aux services de l'État, n'utilise presque pas cette désignation (2\%), visiblement réservée aux supports de communication diffusés dans le bassin houiller ("grisou » y représente 40,8\% des désignations utilisées).

Un dernier document se distingue des précédents. Il s'agit d'une étude produite par une équipe interdisciplinaire de chercheurs universitaires, sous la direction d'un géologue, et publiée sous le titre Le projet d'exploitation du gaz de charbon en Lorraine et son intégration dans le territoire (Gunzburger éd., 2016). La vocation scientifique de ce «livre vert» (ainsi désigné par l'équipe de chercheurs) est affirmée - tout comme sa neutralité dans le débat - par les contributeurs rencontrés lors d'entretiens en 2017. Il importe tout de même de souligner que ce "projet fédérateur», comme le laboratoire de géosciences qui le porte, est financé par des fonds publics accordés pour le soutien de ce laboratoire au «développement des champs gaziers lorrains » (convention de financement région Grand Est / université de Lorraine / Française de l’Énergie, 13 juillet 2017). Ajoutons que plusieurs des contributeurs ne sont pas spécialistes des hydrocarbures, mais issus des sciences humaines, et que toute l'équipe a collaboré étroitement avec l'opérateur gazier.

Dans cette étude, plus encore que dans les trois autres, les désignations employées sont variées; elles diffèrent selon les contributeurs, et varient souvent au sein d'une même contribution. Les termes plurivoques utilisés par les acteurs techno-industriels y dominent pourtant nettement. Dans l'ensemble du document (130 pages), on relève, pour désigner le même gaz : gaz de charbon (51,9 \% des occurrences) ; gaz de houille (18,4\%); CBM (17,9\%); grisou $(5,7 \%)$. En outre, des glissements de sens et des usages fautifs des termes s'observent dans plusieurs contributions. Par exemple, une contribution intitulée 
«L'exploitation du gaz de charbon en Allemagne» traite presque exclusivement de gaz de mine, avec un cadrage initial incitant à confondre les deux types d'extraction :

En Allemagne, la récupération et l'utilisation du gaz de charbon a déjà une longue tradition, puisque les premiers essais dans des exploitations en activité remontent à 1908 dans le bassin houiller de la Sarre. Il existe actuellement, dans ce bassin, un réseau de près de $100 \mathrm{~km}$ de longueur pour alimenter en gaz de charbon différentes installations industrielles [...]. (Deshaies, 2016, p.110)

L'auteur décrit, sous le vocable «gaz de charbon»... le gaz de mine allemand, objet de la quasi-totalité de sa contribution. La comparaison paraît donc inadaptée pour «mettre en perspective» le projet lorrain de CBM et conclure à une acceptation sociale probablement aussi bonne en France qu'en Allemagne. Une autre contribution, qui rend compte d'une quinzaine d'interviews d'anciens mineurs menées conjointement par un historien, un géographe et deux psychologues sociales pour «mettre en évidence le discours des anciens mineurs sur l'acceptabilité sociale de cette [...] éventuelle exploitation future » (Raggi, 2016, p. 49), mobilise grisou, terme visiblement utilisé dans le guide d'entretien :

Le grisou était l'ennemi du mineur. La possibilité de son exploitation à des fins énergétiques peut donc entraîner un changement radical de son image : "C'est du gaz qui nous a embêté [sic] pendant des années et des années, si on peut l'utiliser pour autre chose et non pas pour faire des victimes, pourquoi pas?» [...] D'autant que la localité de Behren-lès-Forbach avait été chauffée en partie grâce au gaz de houille jusqu'en 2009. (Raggi, 2016, p.50; nous soulignons)

La mention répétée de la dangerosité de ce gaz, la reprise littérale de la formule matrice de la communication de l'entreprise («l'ennemi du mineur»), la mention du chauffage de Behren-lès-Forbach par du gaz qui était en réalité du gaz de mine montrent que l'enquête présentée induisait nécessairement les répondants en erreur, puisqu'elle les interrogeait sur le captage de grisou (gaz de mine), et non de CBM. La conclusion de l'article est donc, sans surprise, que les anciens mineurs sont plutôt favorables au projet de la société gazière! Interrogés, plusieurs des auteurs de cette enquête, issus des sciences sociales, disent ne pas distinguer nécessairement entre ces différents gaz, et avoir préféré laisser aux géologues des explicitations terminologiques pour lesquelles ils ne s'estiment pas légitimes. D’autres contributeurs de ce rapport développent la même justification.

Il n'est évidemment pas question d'accuser de manipulation ces chercheurs, probablement simplement soucieux de ne pas sortir de leur champ de compétences, peut-être aussi mal informés par les géologues pilotes du travail collaboratif; mais l'analyse des désignations utilisées dans Le projet d'exploitation du gaz de charbon révèle tout de même un cadrage par le flou très favorable au développement du CBM en Lorraine. Il paraît dès lors légi- 
time de s'interroger sur des discours présentés comme relevant de la rationalité scientifique, mais qui fonctionnent comme des relais de la communication de l'entreprise sans apporter une expertise indépendante par rapport à ce discours corporate.

Le risque d'une inféodation de la recherche universitaire aux intérêts économiques et politiques a déjà été souligné, notamment par Nadine Souchard et Yves Bonny :

À l'heure de la technoscience, de l'économie dite de la connaissance, de la recherche massivement finalisée et orientée par des politiques publiques en tous genres, des incitations croissantes à développer des recherches partenariales avec les entreprises ou la "société civile», continuer à entretenir le mythe d'une vérité scientifique poursuivie exclusivement pour elle-même, dans un sanctuaire institutionnel à l'abri des bruits du monde et des pressions de toutes natures, n'est pas tenable. (Souchard, Bonny, 2015, p.11)

Au-delà des problèmes éthiques que pose une telle inféodation, il paraît légitime de s’interroger sur la visée de telles expertises, en mobilisant les catégories qu'utilise notamment Grégoire Lits dans son article dédié à l'analyse du rôle des chercheurs en sciences sociales dans la gestion des déchets radioactifs (Lits, 2013) : assument-ils un rôle d'informateur ou de médiateur? Dans le cas du CBM, interviennent-ils dans le débat pour l'éclairer ou pour faciliter l'acceptation du projet de l'entreprise, en relayant sa communication d'acceptabilité et en lui cherchant des cautions scientifiques? C'est ce que laisse penser le document de promotion de l'étude Le projet d'exploitation du gaz de charbon en Lorraine et son intégration dans le territoire, document qui reprend textuellement les éléments de langage de l'entreprise - «Le gaz de charbon (grisou), ennemi du mineur hier, peut redonnervie à un bassin minier en voie de paupérisation » - en y ajoutant, en gros caractères, cette annonce: «La caution de scientifiques toutes disciplines »7. Le flou des désignations employées par cette équipe pluridisciplinaire, et la représentation exclusivement positive qu'elles construisent montrent que l'acte de nomination scientifique n'est pas exempt d'enjeux sociopolitiques.

Les enjeux de la nomination dans la controverse autour du CBM sont de différents ordres. D’ordre technique, d'abord, puisque nommer, dans ce cas, c'est inclure l'hydrocarbure controversé dans des classes : celle des énergies indigènes (le charbon) ou au contraire exogènes (les hydrocarbures de rochemère) ; celle des énergies faciles d'accès (conventionnelles) ou au contraire difficiles à extraire (non conventionnelles); et, en définitive, celle des énergies fossiles ou celle des «énergies vertes» - autant de choix déterminants pour

7. «Exploiter le charbon lorrain. Le point de vue des chercheurs », Lettre d'information du laboratoire GeoRessources, n 7 , juin 2018, http://georessources.univ-lorraine.fr/sites/georessources.univ-lorraine.fr/files/users/documents/newsletter7_4_3.pdf (consulté le 21/11/2018). 
l'acceptation sociale de la filière. D'ordre politique, aussi, puisque le choix de certains termes a le pouvoir d'escamoter le débat public en désinformant des populations particulièrement vulnérables à un usage affectif et mémoriel du lexique. Celui-ci constitue donc un enjeu fort pour les acteurs respectifs, afin d'imposer dans l'espace public des désignations axiologiquement marquées et ainsi peser sur les représentations de cet hydrocarbure, pour éluder ou au contraire souligner ses impacts environnementaux, et donc pour étouffer ou aviver les contestations. De fait, une enquête de terrain que nous avons menée en octobre 2017 dans une commune concernée par des forages montre que la stratégie du brouillage, qui consiste à multiplier des désignations sans les affecter de manière continue et cohérente à des référents stables, neutralise en partie les oppositions : $44 \%$ des personnes interrogées déclarent ne pas savoir quel est le gaz recherché par EGL, et plus des deux tiers de ces personnes se disent «sans opinion » face aux forages en raison, justement, de leur ignorance.

Selon nous, et sans cacher que cette qualification relève d'une conception normative de la communication, un tel usage de la langue relève de la manipulation, répondant à des critères notamment définis par Philippe Breton (1997) : manipulation des affects; manipulation des faits par le mélange subtil entre vraies et fausses informations; manipulation cognitive enfin, puisque ces discours suggèrent sans les démontrer des rapports entre des énergies totalement différentes par leurs techniques d'extraction et leurs impacts environnementaux. Qu'une entreprise défende ses activités en usant de telles méthodes est bien connu; que la presse relaie fidèlement ce discours stratégique n'a rien d'exceptionnel. En revanche, que des experts affirmant leur neutralité face au bien-fondé d'une future exploitation du Coal Bed Methane en France adoptent les choix sémantiques des promoteurs techno-industriels, interroge. En soi, l'implication du chercheur ne pose pas problème. Le problème réside plutôt dans la mise en scène d'une posture objective et surplombante, qui justifierait que les experts soient au-dessus du débat et aient le dernier mot. «Parole à la science » était d'ailleurs l'intitulé d'une soirée de présentation du rapport étudié plus haut. Cela signifie-t-il «Silence aux citoyens » ? Surtout, cette apparente objectivité scientifique trompe les récepteurs de l’information livrée, séduits par l'ethos du chercheur spécialiste, neutre, rationnel.

Cet «art de gouverner une technologie contestée » grâce au contrôle systématique des mots illustre le propos de S. Topçu, qui voit dans le langage un instrument extraordinaire pour «construire, porter, voire imposer un imaginaire relatif à des innovations techniques », constituant en cela un instrument majeur de gouvernementalité (Topçu, 2013, p. 196). Malgré l'efficacité politique du procédé, l’imposition dans le débat public d'une terminologie qui tronque la réalité et convoque un imaginaire sans lien réel avec la technologie débattue appelle nécessairement à un questionnement éthique, puisqu'elle enlève aux citoyens, en la matière, la possibilité d'un exercice éclairé de leur citoyenneté. 


\section{Références}

Amossy Ruth, Koren Roselyne, 2010, «Argumentation et discours politique», Mots. Les langages du politique, $\mathrm{n}^{\circ}$ 94, p. 13-21.

ANGENOT Marc, 2014, "La rhétorique de la qualification et les controverses d'étiquetage », Argumentation et analyse du discours, $\mathrm{n}^{0}{ }_{13}$, https://journals.openedition. org/aad/1787 (consulté le 21 novembre 2018).

Bonijoly Didier, Didier Christophe, FABriol Hubert, 2013, Synthèse sur les gaz de houille : exploitation, risques et impacts environnementaux. Rapport final, INERIS, BRGM, MEDDE, http://www.brgm.fr/sites/default/files/brgm-ineris_rapport-gazhouille_2013.pdf (consulté le 21 mars 2018).

BRETON Philippe, 1997, La parole manipulée, Paris, La Découverte.

CGIET, CGEDD, 2012, Les hydrocarbures de roche-mère en France, https://www.ladocumentationfrancaise.fr/var/storage/rapports-publics/124000163.pdf (consulté le 21 mars 2018).

Challleux Sébastien, 2015, "Non au gaz de schiste! Cadrages et débordements de la controverse sur les hydrocarbures non conventionnels en France et au Québec, thèse sous la direction de L. Guay et A. Roger, Université Laval (Québec) et IEP de Bordeaux, https://tel.archives-ouvertes.fr/tel-01280729 (consulté le 12 avril 2018).

Chateauraynaud Francis, Debaz Josquin, 2011, «L'affaire des gaz de schiste. Anatomie d'une mobilisation fulgurante », Socio-informatique et argumentation, https:// socioargu.hypotheses.org/3262 (consulté le 5 octobre 2018).

Deshales Michel, 2016, «L'exploitation du gaz de charbon en Allemagne », dans Le projet d'exploitation du gaz de charbon en Lorraine et son intégration dans le territoire, rapport final (21 juillet 2016), Y. Gunzburger éd., p. 110-116, https://www.francaisedelenergie.fr/medias/pdf/livrevertgazhouille.pdf (consulté le 12 avril 2018).

FORTIN Marie-José, FournIS Yann, 2014, «Vers une définition ascendante de l'acceptabilité sociale : les dynamiques territoriales face aux projets énergétiques au Québec », Natures Sciences Sociétés, vol. XXII, n³ 3, p. 231-239.

FREUND Andreas, 1991, Journalisme et mésinformation, Grenoble, La Pensée sauvage.

Gendron Corinne, 2014, "Penser l'acceptabilité sociale : au-delà de l'intérêt, les valeurs », Communiquer, $\mathrm{n}^{\circ} 11, \mathrm{p} .117-129$.

GUNZBURGER Yann éd., 2016, Le projet d'exploitation du gaz de charbon en Lorraine et son intégration dans le territoire, rapport final (21 juillet 2016), https://www.francaisedelenergie.fr/medias/pdf/livrevertgazhouille.pdf (consulté le 12 avril 2018).

KOREN Roselyne, 2016, «Introduction », Argumentation et analyse du discours, n 17 , https://journals.openedition.org/aad/2295 (consulté le 21 novembre 2018).

KRIEg-Planque Alice, 2010, "La formule "développement durable" : un opérateur de neutralisation de la conflictualité », Langage et société, nº 134, p.5-29.

- 2000, "La dénomination comme engagement: débats dans l'espace public sur le nom des camps découverts en Bosnie », Langage et société, nº 93, p.33-69.

LECOLLE Michelle, 2007, «Enjeux argumentatifs de la nomination : le cas du nom collectif communauté dans les discours publics contemporains", dans Argumentation, manipulation, persuasion, C. Boix éd., Paris, L'Harmattan, p. 227-247.

LENOIR Jean-Claude, BATAILLE Christian, 2013, Rapport sur les techniques alternatives à la fracturation hydraulique pour l'exploration et l'exploitation des hydrocarbures 
non conventionnels, Office parlementaire d'évaluation des choix scientifiques et technologiques, https://www.senat.fr/rap/r13-174/r13-1741.pdf (consulté le 21 novembre 2018).

LIBAERT Thierry, 2009, "Aléa communicationnel et communication sensible : pour une reconnaissance de l'effet d'incertitude appliquée à la communication d'entreprise », Communication \& Organisation, nº36, p.150-177.

LITS Grégoire, 2013, «Analyse du rôle des chercheurs en sciences sociales dans la gestion des déchets radioactifs ", [VertigO]. La revue électronique en sciences de l'environnement, vol. XIII, $\mathrm{n}^{0} 2$, https://journals.openedition.org/vertigo/14207 (consulté le 21 octobre 2018).

MAISONNIER Guy, 2008, «CBM : bilan et perspectives», dans Panorama 2008, Institut français du pétrole, http://www.ifpenergiesnouvelles.fr/Publications/Analysestechnico-economiques/Notes-de-synthese-Panorama/Panorama-2008/CBM-bilanet-perspectives (consulté le 12 avril 2018).

RAGGI Pascal, 2016, "Les ex-“gueules noires” et la perspective d'une exploitation du gaz de charbon en Lorraine», dans Le projet d'exploitation du gaz de charbon en Lorraine et son intégration dans le territoire, rapport final (21 juillet 2016), Y. Gunzburger éd., p. 48-53, https://www.francaisedelenergie.fr/medias/pdf/livrevertgazhouille. pdf (consulté le 12 avril 2018).

SouCHARD Nadine, BonnYYves, 2015, "La recherche-action coopérative, une voie contributive aux productions de la société civile», dans Actes du Colloque «Chercheur.e.s et acteur.e.s de la participation : liaisons dangereuses et relations fructueuses», http://www.participation-et-democratie.fr/sites/default/files/article_souchardbonny_mars_2015.pdf (consulté le 29 mars 2018).

Topçu Sezin, 2013, La France nucléaire : l'art de gouverner une technologie contestée, Paris, Seuil.

Vially Roland, MAISONNIER Guy, RouAud Thierry, 2013, Hydrocarbures de roche-mère : état des lieux, IFPÉnergies nouvelles, http://www.ufip.fr/documents/ajax/les-hydrocarbures-de-roche-mere-etat-des-lieux-ifpen (consulté le 12 mars 2018).

\section{Résumé / Abstract / Compendio}

\section{La controverse du «gaz de charbon » en France (2006-2018) : conflits de nomination et mise en question de la neutralité de l'expertise}

Depuis plusieurs années se développe dans l'est et le nord de la France une controverse autour de forages exploratoires de "gaz de couche de charbon», également désigné comme "gaz de couche», " de houille», puis « de charbon », et même comme " grisou». Ces désignations, évolutives et non stabilisées, sont au cœur d'une vive «controverse d'étiquetage» entre "pros» et "antis», qui parcourt aussi bien les discours des différentes parties prenantes de cette controverse que les textes de presse et les rapports institutionnels, techniques ou scientifiques sur le sujet. L'analyse de ce conflit de nomination évolutif montre qu'il vise, pour les acteurs respectifs, à imposer dans l'espace public une dénomination axiologiquement marquée de cet hydrocarbure afin de peser sur les représentations de son exploitation. Cet article décrit les fonctionnements et les enjeux argumentatifs, stratégiques et sociopolitiques de ces désignations, 
par une approche à la fois lexicologique et rhétorique qui permet de préciser les axiologies à l'œuvre derrière les choix de dénomination, jusque dans le discours d'expertise.

Mots-clés : controverse, désignations, lexique, désinformation, expertise, hydrocarbures

\section{The controversy over "coal gas" in France (2006-2018): naming conflicts and the questioning of expertise neutrality}

For several years, a controversy has developed in Eastern and Northern France over the exploratory drilling of "layer coal gas", also referred to as "layer gas", "coal gas" and even "firedamp". The terms are evolving and non-stabilised and have been at the heart of a "labeling controversy" between their advocates and detractors. The controversy runs through the discourse of the protagonists, through press articles and institutional, technical and scientific reports. In analyzing a naming conflict that is still in the making, this article shows that its actors aim to impose terms that are fraught with value judgement in the public space, in order to weigh on the representation of that fossil fuel's exploitation. It describes the workings and the argumentative, strategic and sociopolitical stakes of the terms, thanks to a lexical and rhetorical examination that pinpoints the value systems underlying naming choices, even in expert discourse.

Keywords: controversy, naming, lexicon, disinformation, expertise, fossil fuels

\section{La controversia del «gas de carbón» en Francia (2006-2018): conflictos de designación y el cuestionamiento de la neutralidad de los conocimientos especializados}

Ya hace muchos años que se desarrolla en el este y en el norte de Francia una controversia relativa a las perforaciones exploratorias del "gas de capa de carbón», igualmente designado como «gas de capa», «de hulla», y más tardiamente «de carbón», y mismo como «grisou». Estas designaciones, evolutivas y no stabilizadas, entan en el centro de una fuerte «controversia de etiquetado» entre "pros»y «antis», que recorre tanto los discuros de los diferentes partidos de esta controversia como los textos de la prensa y los informes institucionales, técnicos o científicos sobre el tema. El analisie de este conflicto de designación evolutivo muestra que apunta, para los actores respectivos, imponer en el espacio público una denominación axiologicamente determinada de este hidrocarburo con el propósito de influir las representaciones sociales relativas a su explotación. Este artículo describe los funcionamientos y lo retos argumentativos, estratégicos y sociopolíticos de estas designaciones, gracias a un enfoque tanto lexicológico como retórico que permite apuntar las axiologías en acción detrás de las opciones de denominación hasta en el discurso de los discursos del experto.

Palabras claves: controversia, designaciones, léxico, desinformación, experto, hidrocarburos 
